\title{
Corrigendum: Analyzing outliers: influential or nuisance?
}

Naomi Altman \& Martin Krzywinski

Nat. Methods 13, 281-282 (2016); published online 30 March 2016; corrected after print 14 April 2016

In the version of this piece initially published, there were two errors. The equation describing mean squared error (MSE) was incorrect in the PDF file. In the legend for Figure 1a, the stated values for mean height and mean weight were switched. The errors have been corrected in the HTML and the PDF versions of the piece. 